Mon. Not. R. Astron. Soc. 000,1177(2020) Printed 5 February $2020 \quad$ (MN L $\mathrm{T}_{\mathrm{E}} \mathrm{X}$ style file v2.2)

\title{
A Shapiro delay detection in the pulsar binary system PSR J1811-2405
}

\author{
C. $\mathrm{Ng}^{1,2 \star}$, L. Guillemot ${ }^{3,4}$, P. C. C. Freire ${ }^{2}$, M. Kramer ${ }^{2,5}$, D. J. Champion ${ }^{2}$, \\ I. Cognard ${ }^{3,4}$, G. Theureau ${ }^{3,4,6}$, E. D. Barr ${ }^{2,7}$ \\ ${ }^{1}$ Dunlap Institute for Astronomy and Astrophysics, University of Toronto, 50 St. George Street, Toronto, ON M5S 3H4, Canada \\ ${ }^{2}$ Max-Planck-Institut für Radioastronomie, Auf dem Hügel 69, D-53121 Bonn, Germany \\ ${ }^{3}$ Laboratoire de Physique et Chimie de l'Environnement et de l'Espace, LPC2E, CNRS-Université d'Orléans, F-45071 Orléans, France \\ ${ }^{4}$ Station de Radioastronomie de Nançay, Observatoire de Paris, CNRS/INSU, F-18330 Nançay, France \\ ${ }^{5}$ Jodrell Bank Centre for Astrophysics, University of Manchester, Alan Turing Building, Oxford Road, Manchester M13 9PL, United Kingdom \\ ${ }^{6}$ LUTH, Observatoire de Paris, PSL Research University, CNRS, Université Paris Diderot, Sorbonne Paris Cité, 92195, Meudon, France \\ ${ }^{7}$ Centre for Astrophysics and Supercomputing, Swinburne University of Technology, Mail H30, PO Box 218, VIC 3122, Australia
}

Released 2020 Xxxxx XX

\begin{abstract}
This paper presents the first detection of Shapiro delay from the binary millisecond pulsar PSR J1811-2405. We report a 11- $\sigma$ measurement of the orthometric amplitude, $h_{3}=$ $6.8(6) \times 10^{-7}$, and a $16-\sigma$ measurement of the orthometric ratio, $\varsigma=0.81(5)$. Given the relatively high orbital inclination, $i=79(2)^{\circ}$, of this binary system, we obtain constraints on the companion mass of $m_{\mathrm{c}}=0.31_{-0.06}^{+0.08} M_{\odot}$. The pulsar mass is currently less well constrained, with a value of $2.0_{-0.5}^{+0.8} M_{\odot}$. The companion mass and the orbital period are in agreement with the prediction made by previous numerical calculations of the evolution of compact binary systems. From a study of the polarization, we find that the orbital inclination angle is $\sim 100^{\circ}$ and that PSR J1811-2405 is an orthogonal rotator. In addition, the $\mu$ s-level timing precision together with its narrow profile make PSR J1811-2405 a good candidate for inclusion in the pulsar timing arrays being used to detect $\mathrm{nHz}$ Gravitational waves.
\end{abstract}

Key words: stars: neutron - pulsars: binaries - pulsars: individual: PSR J1811-2405 .

\section{INTRODUCTION}

PSR J1811-2405 was discovered by the High Timing Resolution Universe (HTRU) Pulsar survey (Keith et al. 2010) conducted with the 64-m Parkes radio telescope. This millisecond pulsar binary system was initially published by Bates et al. (2011). PSR J1811-2405 was reported to be a typical low-mass binary pulsar likely with a Helium white dwarf (He-WD) companion in an orbit of roughly $6.27 \mathrm{~d}$. No post-Keplerian (PK) parameters were detected at the time of the previous publication.

Using the best available radio timing ephemeris, $\mathrm{Ng}$ et al. (2014) detected gamma-ray pulsations from this pulsar in data from the Fermi Gamma-ray Space Telescope (Fermi; Atwood et al. 2009). In order to keep the radio ephemeris up to date and allow for accurate folding of all the gamma-ray data, this pulsar is observed regularly with the Nançay Radio Telescope. Thanks to this as well as a dedicated timing campaign with the Effelsberg Radio Telescope, we have timing data spanning a total of $6.9 \mathrm{yr}$. As a result of this timing project, we have detected the Shapiro delay for this system, and showed that it has a fairly edge-on orbital inclination.

First proposed by Shapiro (1964), the Shapiro delay is the retardation in the arrival times of a pulsar's pulses as they propagate through the curved space-time in the close vicinity of the binary companion. This effect is most readily observed in binary systems viewed nearly edge-on, and in some cases allows for precise measurements of the pulsar and companion masses, e.g. PSR J1614-2230 (Demorest et al. 2010). This and PSRs J0348+0432 and J0740+6620, with implied masses of $1.908 \pm 0.016,2.01 \pm 0.04$ and $2.14 \pm 0.10 M_{\odot}$ respectively (Arzoumanian et al. 2018; Antoniadis et al. 2013, Cromartie et al. 2019), have effectively ruled out a number of equations of state for matter at densities above those of the atomic nucleus (EOS), including almost all currently proposed hyperon or boson condensate EOSs. This represents a very important constraint on the unknown state of matter at those densities (Özel \& Freire 2016). Nonetheless, precise neutron star mass measurements in general are hard to achieve because suitable systems are rare: Thus far, only 43 such measurements have been made 1

It is thus of great interest to obtain more neutron star mass measurements, in order to probe a wider parameter space of the

^ E-mail: cherry.ng@dunlap.utoronto.ca

1 http://www3.mpifr-bonn.mpg.de/staff/pfreire/NS_masses.html 
Table 1. Specifications of the observing systems employed for the timing observations in this work. $f_{\mathrm{c}}$ represents the associated receiver central frequency and $B$ is the backend usable bandwidth both in $\mathrm{MHz}$. The number of TOAs generated from each data set is listed in the last column.

\begin{tabular}{llll}
\hline Backend & Receiver $f_{\mathrm{c}}(\mathrm{MHz})$ & $B(\mathrm{MHz})$ & No. TOAs \\
\hline Effelsberg PSRIX & 1347 & 200 & 90 \\
Nançay NUPPI & 1484 & 512 & 2368 \\
Nançay NUPPI & $\sim 2200$ & 512 & 74 \\
\hline
\end{tabular}

EOS and to better understand the true distribution of neutron star masses.

This paper is organized as follows: in Section 2 we describe the radio timing observations conducted for PSR J1811-2405, including the instrumental set-up and the derivation of times-ofarrival. In Section 3 we present our updated timing solution and discuss results from the polarization study as well as from the Bayesian $\chi^{2}$ analysis of the masses of the pulsar and its companion. We summarise our work in Section 4

\section{RADIO TIMING OBSERVATIONS}

\subsection{Observational set-up}

Observations at the Nançay Radio Telescope (NRT) began in 2012 as part of their regular gamma-ray pulsar follow-up timing campaign. The Nançay Ultimate Pulsar Processing Instrument (NUPPI; Desvignes et al. 2011) was employed to take coherently dedispersed timing data. The NUPPI data set has high timing precision and is the longest span data set we have.

Despite its high instrumental sensitivity, the meridian nature of the NRT meant that PSR J1811-2405 could not be tracked for more than an hour. Hence we conducted an intense timing campaign with daily observations during the $6.27 \mathrm{~d}$ orbit with the Effelsberg 100-m Radio Telescope in November 2015. This campaign was designed to track the pulsar as long as possible on November 19 during superior conjunction. Shorter tracks were taken either side of the superior conjunction observation to ensure good orbital coverage and to increase the confidence level of the Shapiro delay measurement. The Effelsberg observations were taken at the 1.4$\mathrm{GHz}$ band using the central feed of the 7-beam receiver (P217mm). We used the PSRIX backend (Lazarus et al. 2016) which is based on the ROACH board and collects data in coherent dedispersion folding mode. One 1.6-hr scan was carried out at a wavelength of $6 \mathrm{~cm}$ using the $560 \mathrm{~mm}$ receiver although no pulsar signal was detected, setting an upper limit of flux density at $S_{4.9 \mathrm{GHz}}<0.02 \mathrm{mJy}$. Refer to Table 1 for the specifications of all the receivers employed in this timing analysis.

\subsection{Derivation of times-of-arrival and timing solution}

The PSRCHIVE data analysis package (Hotan et al.|2004) was used for the radio timing data reduction. Each observation was corrected for dispersion and folded at the predicted topocentric pulse period. We co-added high signal-to-noise observations and created noise-free reference template for each observing frequency using wavelet smoothing as implemented in the psrchive program of psrsmooth (Demorest et al. 2015). We convolved the template with each individual profile to produce a time-of-arrival (TOA) (Taylor 1992). Multiple TOAs per observation were generated when possible, by downsampling the bandwidth to four fre-
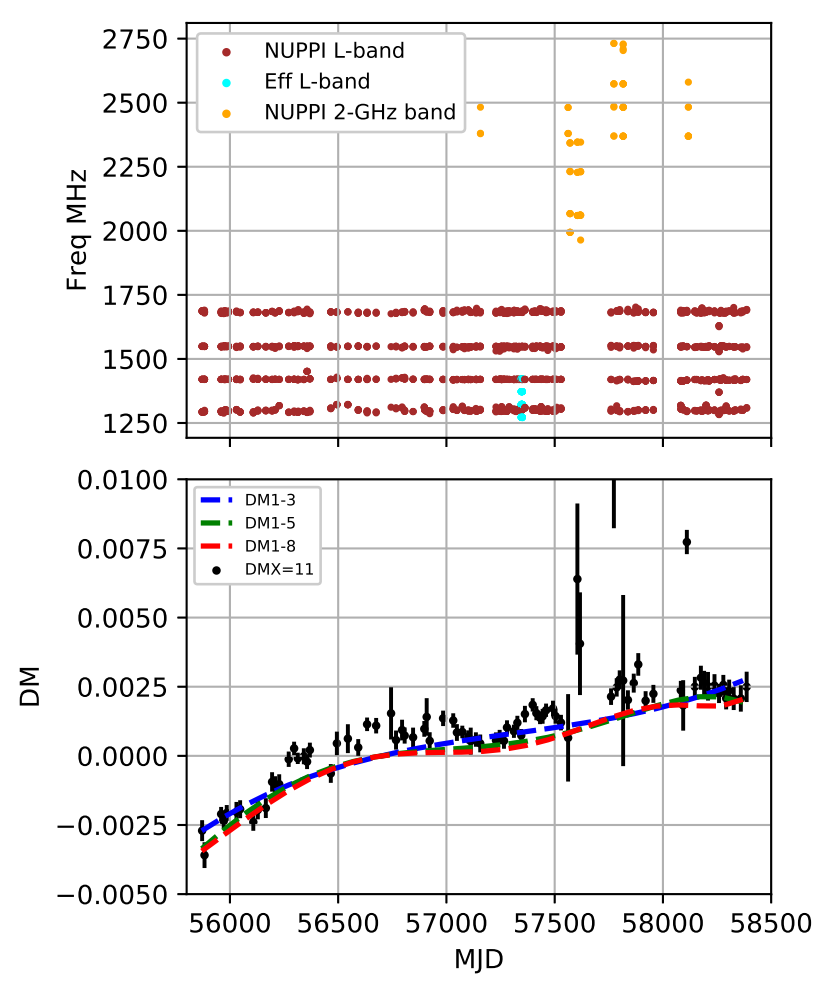

Figure 1. Top panel: the temporal coverage of observing frequency of the coherently dedispersed timing data used in this analysis. We create four sub-bands per observation when possible. The plot is colour-coded to show the different telescopes and backends, including Nançay NUPPI at L-band (brown), Nançay NUPPI at $2 \mathrm{GHz}$ (orange) and Effelsberg PSRIX (cyan). Bottom panel: DM variation as a function of time, measured by fitting for DMX with a window of $11 \mathrm{~d}$ (black) in TEMPO2. There are small timescale DM variations that cannot be modeled properly using DM derivatives (blue: up to the third derivatives; green: up to the 5th derivative; red: up to the 8 th derivative).

quencies and summing over every $10 \mathrm{~min}$. This is appropriate for the accurate measurement of orbital effects such as Shapiro delay for a 6-d binary like PSR J1811-2405. The DE421 Solar System ephemeris of the Jet Propulsion Laboratory (Folkner et al. 2009) was used to transform the TOAs to the Solar System barycentre. Finally, the TEMPO2 software package (Hobbs et al.2006) was used to fit a timing model to all TOAs, taking into account the astrometry, spin, and orbital motion of the pulsar.

As mentioned in $\mathrm{Ng}$ et al. (2014), PSR J1811-2405 lies very close to the ecliptic plane with ecliptic coordinates $(\lambda, \beta)=$ $\left(272^{\circ} .586,-0^{\circ} .675\right)$. With a $\lambda$ so close to $270^{\circ}$, the uncertainty in $\beta$ will only pertain to the declination when transforming in equatorial coordinates. We hence fixed proper motion in declination at zero for the rest of the analysis. The parallax was also fixed at zero as the timing precision of our data is not high enough for measuring parallax with significance.

A careful modelling of the Dispersion Measure (DM) is very important for this work because any unaccounted temporal DM variations can degrade the precision of a Shapiro delay measurement. The fact that PSR J1811-2405 is on the ecliptic plane also means it is more susceptible to solar wind DM contribution. We used the solar wind model implemented by default in TEMPO2 (where the density of electrons is proportional to $1 / r_{\odot}^{2}$, with $r_{\odot}$ being the distance to the Sun, see Edwards, Hobbs \& Manchester 

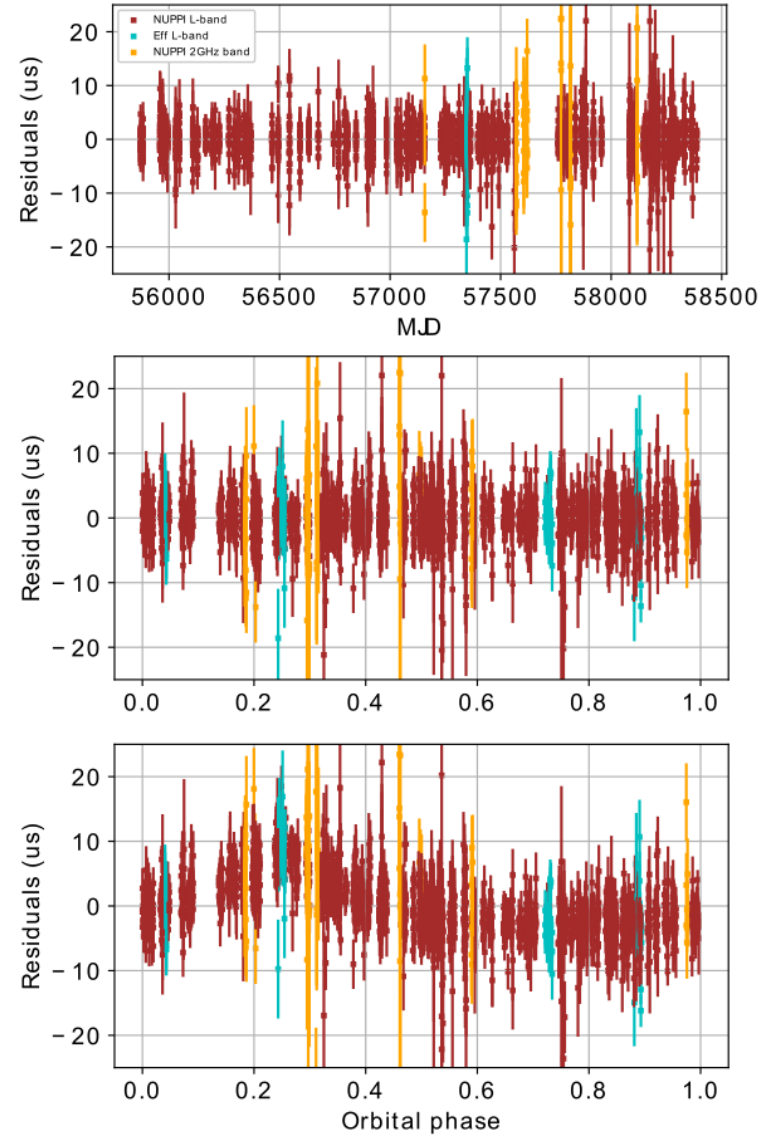

Figure 2. Timing residuals for PSR J1811-2405 as a function of MJD (top) and orbital phase (middle, bottom). TOAs are colour-coded to show different telescopes and backends, including Nançay NUPPI at L-band (brown), Nançay NUPPI at $2 \mathrm{GHz}$ (orange) and Effelsberg PSRIX (cyan). 20 CM (blue), Parkes 50 CM (cyan). The middle panel shows the best-fit residuals when all parameters including the Shapiro delay are fitted for. The bottom panel shows the residuals if $\sin i$ and $m_{\mathrm{C}}$ are set to zero and all other parameters fixed at their best fit values. The expected characteristic signature can be seen at orbital phase 0.25 . The error bars represent the $1-\sigma$ uncertainties of the TOA measurements.

2006). But instead of the default value of 4 electrons $/ \mathrm{cm}^{3}$ at the Earth's distance from the Sun proposed by Edwards, Hobbs \& Manchester (2006), we used 5 electrons $/ \mathrm{cm}^{3}$, which was found to yield slightly better results by Arzoumanian et al. (2018). The timing baseline of PSR J1811-2405 coincides in time with the NANOGrav timing baseline, thus warranting the use of the same electron model. We disregarded any observations that were taken with a solar elongation angle $<15^{\circ}$.

This simple model is not enough to describe DM variations from the Solar wind, and it certainly cannot describe DM variations caused by the ionised interstellar medium. In order to do that, we must in addition use the DMX parameterization in the TEMPO2 software package. This a piecewise linear fit of temporal DM variations (see NANOGrav Collaboration et al. 2015 for a detailed definition). It can be used to measure DM variations with a bin size of several days. In the bottom panel of Fig. 1 we used a 11-d DMX window and showed that there is a temporal DM variation of the order of $0.005 \mathrm{~cm}^{-3} \mathrm{pc}$ over the course of our timing data.
Table 2. TEMPO 2 best-fit parameters for PSR J1811-2405. Values in parentheses are the nominal $1-\sigma$ uncertainties in the last digits.

\begin{tabular}{|c|c|}
\hline \multicolumn{2}{|c|}{ Spin and astrometric parameters } \\
\hline Right ascension, $\alpha(\mathrm{J} 2000)$ & $18: 11: 19.854050(19)$ \\
\hline Declination, $\delta(\mathrm{J} 2000)$ & $-24: 05: 18.422(10)$ \\
\hline Proper motion in R.A., $\mu_{\alpha}\left({\left.\operatorname{mas~} \mathrm{yr}^{-1}\right)}^{-1}\right.$ & $0.53(6)$ \\
\hline Spin period, $P(\mathrm{~ms})$ & $2.66059327687744(2)$ \\
\hline Period derivative, $\dot{P}$ & $1.33756(3) \times 10^{-20}$ \\
\hline Dispersion measure, $\mathrm{DM}\left(\mathrm{cm}^{-3} \mathrm{pc}\right)$ & $60.6196(2)$ \\
\hline Rotation measure $\left(\mathrm{rad} \mathrm{m}^{-2}\right)$ & $21(9)$ \\
\hline \multicolumn{2}{|l|}{ Binary parameters } \\
\hline Orbital model & ELL1H \\
\hline Orbital period, $P_{\text {orb }}$ (days) & $6.27230196915(11)$ \\
\hline Projected semi-major axis, $x$ (lt-s) & $5.7056569(8)$ \\
\hline Epoch of ascending node, $T_{\text {asc }}(\mathrm{MJD})$ & $56327.90581153(2)$ \\
\hline$e \sin \omega, \epsilon_{1}$ & $9.9(9) \times 10^{-7}$ \\
\hline$e \cos \omega, \epsilon_{2}$ & $5.0(3) \times 10^{-7}$ \\
\hline Orthometric amplitude, $h_{3}(\mu \mathrm{s})$ & $0.68(6)$ \\
\hline Orthometric ratio, $\varsigma$ & $0.81(4)$ \\
\hline \multicolumn{2}{|l|}{ Derived parameters } \\
\hline Inferred eccentricity, $e$ & $1.11(8) \times 10^{-6}$ \\
\hline Longitude of periastron, $\omega\left(^{\circ}\right)$ & $62(2)$ \\
\hline Mass function, $f\left(M_{\odot}\right)$ & $0.00506927(2)$ \\
\hline Companion mass, $m_{\mathrm{c}}\left(M_{\odot}\right)$ & $0.26(6)$ \\
\hline $\sin i$ & $0.978(12)$ \\
\hline Orbital inclination from Bayesian analysis, $i\left(^{\circ}\right)$ & $76.2_{-3.2}^{+2.8}$ \\
\hline $\begin{array}{l}\text { Companion mass from Bayesian analysis, } m_{\mathrm{c}} \\
\left(M_{\odot}\right)\end{array}$ & $0.31_{-0.06}^{+0.08}$ \\
\hline Pulsar mass from Bayesian analysis, $m_{\mathrm{p}}\left(M_{\odot}\right)$ & $2.0_{-0.5}^{+0.8}$ \\
\hline DM-derived distance $(\mathrm{kpc})^{\dagger}$ & 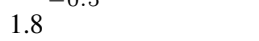 \\
\hline Intrinsic period derivative, $\dot{P}_{\text {int }}$ & $1.284(15) \times 10^{-20 *}$ \\
\hline Characteristic age, $\tau_{\mathrm{r}}(\mathrm{Gyr})$ & 3.15 \\
\hline $\begin{array}{l}\text { Characteristic dipole surface magnetic field } \\
\text { strength at equator, } B_{\text {eq }}\left(10^{8} \mathrm{G}\right)\end{array}$ & 1.9 \\
\hline Spin down power, $\dot{E}\left(10^{34} \mathrm{erg} \mathrm{s}^{-1}\right)$ & 2.7 \\
\hline \multicolumn{2}{|l|}{ Timing model } \\
\hline Binary model & ELL1 \\
\hline Solar System ephemeris & DE421 \\
\hline Timing epoch (MJD) & 56330 \\
\hline First TOA (MJD) & 55871.6 \\
\hline Last TOA (MJD) & 58386.9 \\
\hline Weighted RMS residuals ( $\mu \mathrm{s})$ & 1.81 \\
\hline Reduced $\chi^{2}$ & 1.3 \\
\hline
\end{tabular}

* We have fixed the unconstrained proper motion in declination $\left(\mu_{\delta}\right)$ at zero because PSR J1811-2405 is very close to the ecliptic plane. The transverse velocity is therefore also not measurable. The derived $\dot{P}_{\text {int }}$ represents an upper limit, obtained limit without correcting for any Shklovskii contribution (Shklovskii 1970) in $\mu_{\delta}$.

Both the NE2001 (Cordes, \& Lazio 2002) and the YMW2016 (Yao et al. 2017) electron density models yield the same distance estimate.

Alternatively, we also attempted to model this DM variation using DM derivatives. However, small timescale variations seen in Fig. 11 meant that even including up to the 8th DM derivative, the model still deviates visibly from the data. We hence concluded that DMX appears to be a more reasonable approximation of the DM trend compared to using DM derivatives. We note that neither DM model has predictive power outside the range of data we have here.

Since PSR J1811-2405 has a very circular orbit with eccentricity of the order of $10^{-6}$, we used the ELL1 and ELL1H orbital models (Lange et al. 2001, Freire \& Wex 2010) to characterize it. In the more commonly used orbital models, like the BT (Blandford \& Teukolsky 1976) and DD (Damour \& Deruelle 1986) models, there is a large covariance between two orbital parame- 
ters, the longitude of periastron $(\omega)$ and the epoch of periastron $\left(\mathrm{T}_{0}\right)$; this becomes extremely large for small orbital eccentricities. The ELL1 and ELL1H models avoid this by replacing those parameters and the orbital eccentricity $e$ by the Laplace-Lagrange parameters $\left(\epsilon_{1}=e \sin \omega\right.$ and $\left.\epsilon_{2}=e \cos \omega\right)$ and the time of ascending node passage $\left(T_{\text {asc }}\right)$, these have very small correlations among themselves. The ELL1 and ELL1H models are approximate, they should only be used when the ignored term (with amplitude $\left.x . e^{2}=7.0 \times 10^{-12} \mathrm{lt}-\mathrm{s}\right)$ is smaller than the timing precision $\left(T_{\mathrm{rms}} / \sqrt{N}=3.6 \times 10^{-8} \mathrm{~s}\right)$; this is certainly the case for PSR J1811-2405. This implies that ignoring that term will have, in this case, no other consequences, in particular it will have no effect on the measurement of the Shapiro delay.

Towards the end of the timing analysis when the reduced $\chi^{2}$ is close to one, we can assume that the timing model provides a reliable fit to the data. As a last step, we compensated for any remaining systematics by calculating backend-specific weighing correction (also known as 'EFAC' in TEMPO2). These coefficients were applied to scale the TOA uncertainties such that the reduced $\chi^{2}$ is unity for each individual data set. This procedure yields more conservative and realistic estimates of the uncertainties in the timing parameters.

The timing solution of PSR J1811-2405, obtained using the ELL1H model, can be found in Table 2 Only coherently dedispersed timing data from Nançay and Effelsberg were employed in the timing analysis because they have the highest precision and represent the most homogeneous data set.

The main new result from the timing is a clear detection of the Shapiro delay. Fig. 2 compares the timing residuals when Shapiro delay is and is not taken into account. The signature sharp peak of a Shapiro delay can be seen clearly around phase 0.25 , where the Earth-pulsar line of sight passes nearest to the companion as defined by the ELL1 and ELL1H binary models.

In the ELL1 model the Shapiro delay is characterized by two post-Keplerian parameters, the range $(r)$ and shape $(s)$ (Damour \& Taylor 1992, Will 1993), where, assuming that General Relativity (GR) is the correct description of gravity, $r=T_{\odot} m_{\mathrm{c}}$ and $s=\sin i$, (here $T_{\odot} \equiv G M_{\odot} c^{-3}=4.9254909476412675 \mu \mathrm{s}$ is the mass of the Sun in units of time). From these two parameters, we obtain $m_{\mathrm{c}}=0.26(6) M_{\odot}$ and $\sin i=0.978(12)$. In the ELL1H model the Shapiro delay is described by two different PK parameters, the orthometric ratio $\varsigma$ and amplitude $h_{3}$. Assuming GR, these are given by $\varsigma=\frac{\sin i}{1+|\cos i|}$ and $h_{3}=T_{\odot} m_{\mathrm{c}} \varsigma^{3}$ (Freire $\&$ Wex 2010). These two parameters have much smaller correlation than $r$ and $s$, and hence provide a better description of the $m_{\mathrm{c}}$ and $i$ constraints derived from the Shapiro delay, particularly when the signal is weak. We report a highly significant $(11-\sigma) h_{3}$ of $6.8(6) \times 10^{-7}$ and a $16-\sigma$ measurement of $\varsigma=0.81(5)$; these yield mass and inclination estimates similar to those derived in the ELL1 model. In Fig. 3 we can see that these two parameters provide a good description of the regions of the $\cos i-m_{\mathrm{c}}$ plane where the system is most likely to be located. A detailed analysis of the mass and inclination constraints is presented in section 3.2

\section{RESULTS AND DISCUSSION}

\subsection{Polarization study}

The Effelsberg timing observations recorded the four Stokes parameters in each frequency channel and thus can be used to study the polarization profile. We polarization calibrated each observation for the differential gain and phase between the feeds with an observation of the noise diode coupled to the receptors in the feeds. We made sure that the polarization calibration is taken adjacent to the targeted pulsar observations. In additional to the Effelsberg data sets, PSR J1811-2405 was also followed-up at the Parkes 64-m radio telescope initially after its discovery. Incoherently-dedispersed Parkes Digital Filterbank systems (DFB) is available at L-band. A few observations have also been taken using the $10 / 50 \mathrm{~cm}$ receiver (Granet et al.2005) which allow for the study of pulsar profile variations across frequencies. The Parkes observations were flux calibrated by using an averaged observation of Hydra A and the Effelsberg observation with the quasar 3C353.

Fig. 4 shows the integrated polarization profiles of PSR J1811-2405 in total intensity, linear and circular polarization. We have arbitrarily aligned the main pulse at phase 0.4 across the three available observing frequencies. Our best $732 \mathrm{MHz}$ profile comes from the co-adding of $4.1 \mathrm{~h}$ of Parkes APSR coherently dedispersed data. Our best L-band profile comes from the co-adding of $14.6 \mathrm{~h}$ of Parkes DFB incoherently dedispersed data. At $3100 \mathrm{MHz}$, a total of $4.7 \mathrm{~h}$ of incoherently-dedispersed Parkes DFB observations are available. We attempted to measure the observed Faraday rotation by fitting the position angle (PA) variations at L-band across the $256 \mathrm{MHz}$ band and obtained a rotation measure (RM) of 21(9) $\mathrm{rad} \mathrm{m}^{-2}$. The profiles shown in Fig. 4 have had their RM corrected.

PSR J1811-2405 has a profile comprising two main components: a main pulse and an interpulse. The interpulse appears roughly 0.45 in phase after the main pulse. We can also see that there is significant profile evolution across observing frequencies, as the strength of the interpulse decreases from being almost as bright as the main pulse at $3100 \mathrm{MHz}$ to being almost unidentifiable at $735 \mathrm{MHz}$. The emission of the main pulse changes handedness in circular polarization which is most obvious at $1369 \mathrm{MHz}$. The interpulse is strongly linearly polarized, especially at $3100 \mathrm{MHz}$. PSR J1811-2405 appears to have higher flux at lower observing frequencies, with a peak flux density of only a few mJy at $3100 \mathrm{MHz}$ but over $60 \mathrm{mJy}$ at $732 \mathrm{MHz}$. Such a steep spectral index is typical of pulsars.

We modeled the observed PA swing according to the Rotating Vector Model (RVM; Radhakrishnan \& Cooke 1969). As detailed in Lorimer \& Kramer (2004), the RVM can in principle provide insights on the magnetic inclination angle, $\alpha$, as well as the viewing angle between the observer and the spin axis, $\zeta$. We followed the procedure of a least-squares fit similar to that described in Berezina et al. (2017), by stepping through a range of $\alpha$ and $\zeta$ while simultaneously minimizing the reference phase $\Phi_{0}$ and the reference position angle $\Psi_{0}$ of the RVM at each grid point. This results in the 1- $\sigma$ $\chi^{2}$ contour shown in Fig. 5 From the system parameters, we can expect PSR J1811-2405 to have undergone an extended recycling process, which not only transferred masses but also orbital angular momentum. As a result of this accretion phase, the spin angular momentum of the pulsar aligned with the orbital angular momentum over time. Hence, in order for the pulsar beam to be visible to a terrestrial observer, the viewing angle $\zeta$ must be roughly consistent with the orbital inclination angle (within the uncertainty of the angular radius of the pulsar beam, $\rho$, c.f. Guillemot \& Tauris 2014). We can thus expect $\zeta \approx i$. From the Shapiro delay measurements, we can only determine $\sin i$, hence both $i$ and $180^{\circ}-i$ are possible. With this in mind, we mark the value of $i$ and $180^{\circ}-i$ from our pulsar timing Shapiro delay on Fig. 5] as two horizontal bands, at $79^{\circ}$ and $101^{\circ}$ respectively. The width of these horizontal bands represents the uncertainty on $i$.

The intersection region of the polarimetry and orbital inclina- 


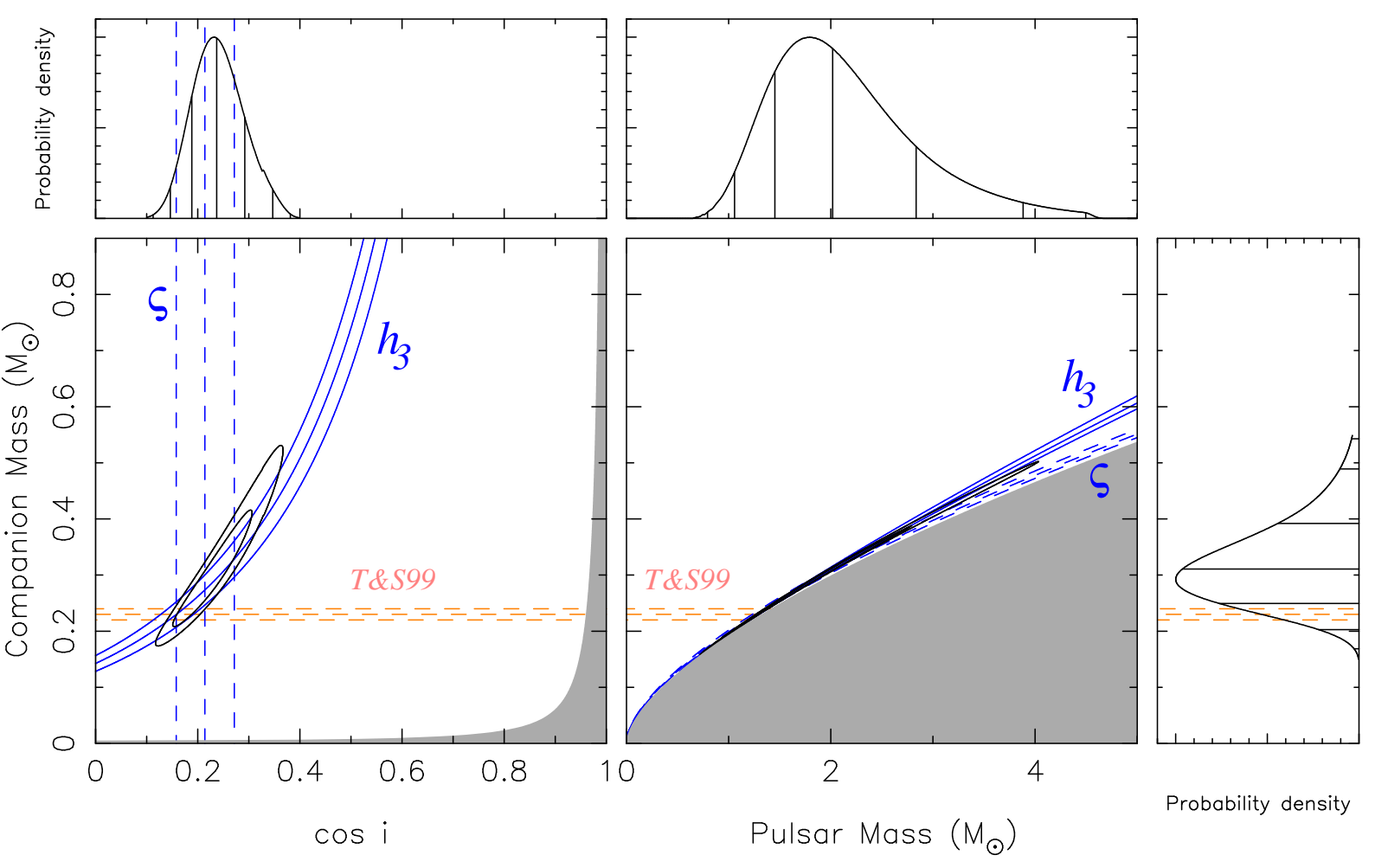

Figure 3. Shapiro delay constraints on the masses and orbital inclination of PSR J1811-2405. The black contours contain 68.27 and 95.45 per cent of the total probabilities of the 2-D pdfs from the Bayesian $\chi^{2}$ analysis. The blue lines correspond to the nominal and $\pm 1-\sigma$ uncertainties associated with the two orthometric parameters, $\varsigma$ (dashed line) and $h_{3}$ (solid line). The pink lines labelled as T\&S99 represent the range of companion masses predicted by Tauris $\&$ Savonije (1999) for the orbital period of this system. Left: $\cos i-m_{\mathrm{c}}$ plane. The gray area is excluded by $m_{\mathrm{p}}>0$. Right: $m_{\mathrm{p}}-m_{\mathrm{c}}$ plane. The gray area is excluded by $\sin i \leqslant 1$. The lateral panels are projected 1-D pdfs of $\cos i, m_{\mathrm{p}}$ and $m_{\mathrm{c}}$ respectively.

tion favours a solution with $\alpha=92^{\circ}$ and $\zeta=79.8^{\circ}$, indicating that the true underlying orbital inclination angle is $i=180^{\circ}-79.8^{\circ} \sim$ $100^{\circ}$. This $\alpha$ value also indicates that PSR J1811-2405 is an orthogonal rotator, which is consistent with the fact that we see an interpulse from the pulse profile. It is sometimes possible to obtain further constraints on $\alpha$ through a fitting of the pulse width, together with an assumption of a filled emission beam. However, the duty cycle of PSR J1811-2405 is relatively low and only the width of the main peak can be measured, which limits the reliability of this analysis. This is not unusual for MSPs, especially when lowlevel components are present as in the case of PSR J1811-2405.

Guillemot \& Tauris (2014) analysed a sample of MSPs with $\zeta$ constraints, and found marginal evidence for different viewing angle distributions between gamma-ray-detected and undetected energy and nearby MSPs. They postulated that gammaray-undetected MSPs are seen under small viewing angles. PSR J1811-2405 is detected in gamma rays and is seen under a large viewing angle, and thus seems to follow the trend. We also compared the gamma-ray profile of PSR J1811-2405 with the light curve models presented in Romani \& Watters (2010). Qualitatively, we found good agreement with their light curve corresponding to $(\alpha, \zeta)=\left(90^{\circ}, 80^{\circ}\right)$. We do not see obvious preference between the 'two pole caustic' (TPC) and the outer gap (OG) model.

\subsection{Pulsar mass constraint from the Shapiro delay measurements}

As described by Splaver et al. (2002), we can estimate the masses and their uncertainties for the pulsar $\left(m_{\mathrm{p}}\right)$ and its companion by performing a Bayesian $\chi^{2}$ analysis in the $m_{\mathrm{c}}-\cos i$ plane. We stepped through a regular grid ranging from $0.15 M_{\odot} \leqslant m_{\mathrm{c}}<$ $0.55 M_{\odot}$ and from $0.1 \leqslant \cos i<0.4$, involving a total of $150 \times 400$ trial values. The assumption of a flat $\cos i$ plane can be justified if we consider the binary orbit to be randomly oriented in space. For each of these $m_{\mathrm{c}}-\cos i$ pairs, we calculated the corresponding $r$ and $s$ to be held fixed. We fit for all other timing parameters and record the final $\chi^{2}$. Fig. 3 shows the resultant $\chi^{2}$ map, from which 2-D probability distribution functions (pdfs) can be derived. The black contours represent the 68.27 and 95.45 per cent of the total probability, which is consistent with that from the fitting of $h_{3}$ and $\varsigma$ (blue lines) using the orthometric parametrization.

We can then marginalize the 2-D pdfs by projecting them onto the $m_{\mathrm{c}}, \cos i$ and $m_{\mathrm{p}}$ axes. From these 1-D pdfs, we obtain $m_{\mathrm{p}}=$ $2.0_{-0.5}^{+0.8} M_{\odot}, m_{\mathrm{c}}=0.31_{-0.06}^{+0.08} M_{\odot}$, and $i=76^{\circ} .2_{-3^{\circ} .2}^{+2^{\circ} .8}$, where the quoted values are the medians together with the $\pm 1-\sigma$ interval. The current value of $m_{\mathrm{p}}$ is not yet very well constrained but could be improved with some more years of pulsar timing.

The detection of other post-Keplerian parameters such as the orbital period variation $\left(\dot{P}_{\text {orb }}\right)$ and the variation in the longitude of periastron $(\dot{\omega})$ could, in a more compact and eccentric system, have helped to narrow down the pulsar mass range and to conduct self-consistency tests of GR and other theories of gravity. For this system, a $\dot{P}_{\text {orb }}$ of the order of $10^{-16}$ is predicted from orbital decay 

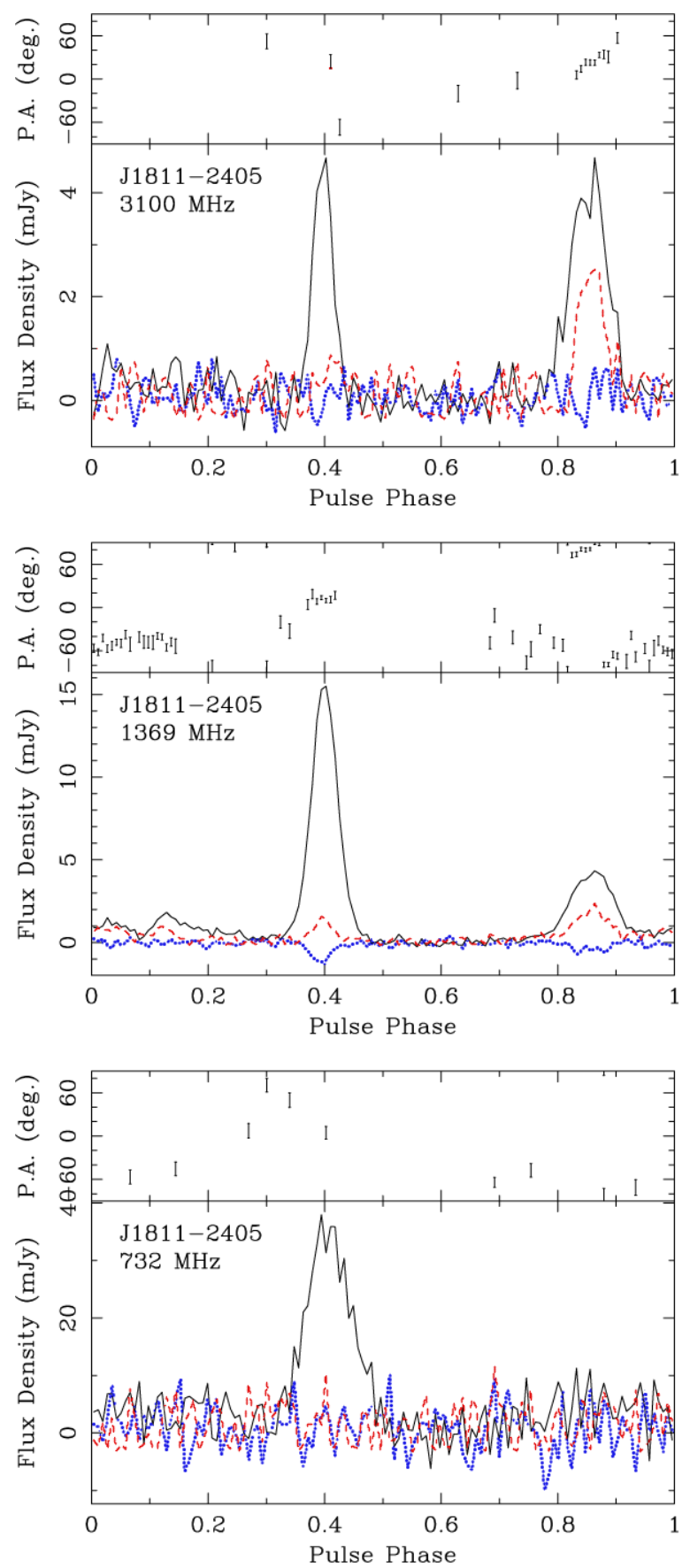

Figure 4. Polarization profiles of PSR J1811-2405 at (top) $3100 \mathrm{MHz}$ from Parkes, (middle) $1369 \mathrm{MHz}$ from Effelsberg and (bottom) $732 \mathrm{MHz}$ from Parkes. The upper panel of each figure shows the RM-corrected PA variation in longitude with respect to the celestial north. Only PAs with signal-tonoise ratios $>3$ are shown. The lower panel shows the integrated profile of total intensity (black solid line), linear polarization (red dashed line) and circular polarization (blue dotted line).

due to GR, which is too small to be easily separated from kinematic effects due to the Galactic acceleration and the Shklovskii effect (Shklovskii 1970), hence we do not expect to measure any intrinsic $P_{\text {orb }}$ in the near future. If we assume a pulsar mass of $1.5 M_{\odot}$, for an orbital inclination of $79^{\circ}$, one can expect an $\dot{\omega}$ of $0.0136^{\circ} \mathrm{yr}^{-1}$. Currently, we measure $\omega$ with a precision of $0.7^{\circ}$. This means we will have to wait at least $50 \mathrm{yr}$ to achieve a $1-\sigma$ measurement of $\dot{\omega}$.
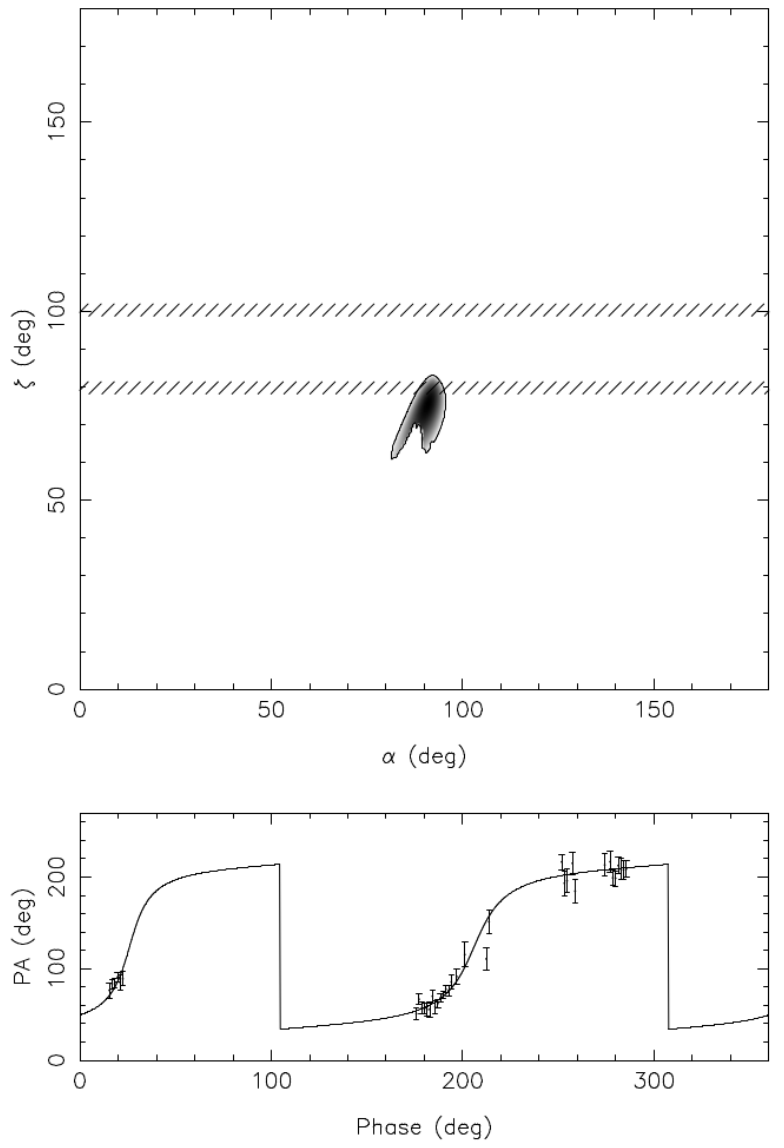

Figure 5. Main panel: System geometry for PSR J1811-2405 from a leastsquares fit of the RVM to the PA. The contour indicates $1-\sigma$ best-fit region of $\alpha$ and $\zeta$. The orbital constraints from our Shapiro delay measurement are marked as two horizontal bands. Bottom panel: The corresponding RVM fit for $\alpha=92^{\circ}$ and $\zeta=79.8^{\circ}$ is shown. An orthogonal shift is applied for the second group of points, as well as a $180^{\circ}$ phase shift between the first and third group.

A more promising improvement can be achieved with the MeerKAT (Bailes et al. 2018) and even better, when the Square Kilometre Array (SKA; Stappers et al.2018) comes online, which will most certainly provide much better constraints on the Shapiro delay measurement.

\subsection{Evolution model}

Tauris \& Savonije (1999) conducted numerical calculations on the non-conservative evolution of close binary systems. They suggested that for diverging LMXBs with a donor mass $<2 M_{\odot}$ and a $1.3 M_{\odot}$ accreting neutron star, if the orbital period is greater than the orbital bifurcation period, i.e. $P_{\text {orb }}>P_{\text {bif }}(\simeq 2$ d), then the system should follow a positive correlation between the orbital period and the mass of the final He-WD companion, quantified by Equation (20) and (21) in Tauris \& Savonije (1999).

PSR J1811-2405 belongs to this type of diverging system, with $P_{\text {orb }}=6.27 \mathrm{~d}$. In Fig. 3. we overplot the range of companion masses predicted by Tauris \& Savonije (1999) for the orbital period of this system. The measurement of $m_{\mathrm{c}}$ is consistent with the prediction of Tauris \& Savonije (1999), but because of its large uncertainties, it cannot yet test the relation. We note that if the com- 
panion has the predicted mass, then the Shapiro delay parameters would imply a pulsar mass around $1.3 M_{\odot}$. It would also mean a $\cos i$ of \pm 0.16 , which equals to $i \sim 81^{\circ}$ or $99^{\circ}$. This is in agreement with the polarization estimate, and matches the orthogonal rotator scenario.

\section{SUMMARY}

We present an updated radio timing solution for the binary system PSR J1811-2405. An extended timing campaign and high precision coherently dedispersed observations have allowed for the first detection of the relativistic Shapiro delay. We measured the orthometric amplitude $h_{3}$ and ratio $\varsigma$ with high significance. By conducting a Bayesian $\chi^{2}$ analysis, we obtained constraints on the companion mass to be $m_{\mathrm{c}}=0.31_{-0.06}^{+0.08} M_{\odot}$ and a less well constrained pulsar mass of $m_{\mathrm{p}}=2.0_{-0.5}^{+0.8} M_{\odot}$.

The companion mass is in agreement with the theoretical $m_{\mathrm{c}}-P_{\text {orb }}$ correlation obtained by Tauris \& Savonije (1999). From the polarization study, we obtained a solution of $\alpha=92^{\circ}$ and $\zeta=79.8^{\circ}$, indicating that the true underlying orbital inclination angle is $i \sim 100^{\circ}$, consistent with PSR J1811-2405 being an orthogonal rotator. The high timing precision and sharp profile of PSR J1811-2405 make it a good candidate to be included in a pulsar timing array to aid the gravitational wave detection effort. With a longer timing baseline we can then expect improvements in the precision of the pulsar mass measurement.

\section{ACKNOWLEDGEMENTS}

The Parkes Observatory is part of the Australia Telescope National Facility, which is funded by the Commonwealth of Australia for operation as a National Facility managed by CSIRO. This work was partly based on observations with the 100-m telescope of the MPIfR (Max-Planck-Institut für Radioastronomie) at Effelsberg. The Nançay radio observatory is operated by the Paris Observatory, associated with the French Centre National de la Recherche Scientifique (CNRS). We also thank Marina Berezina, Eleni Graikou and Gregory Desvignes for advice on Effelsberg data calibration, as well as Andrew Cameron for carefully reading the manuscript.

\section{REFERENCES}

Antoniadis J. et al., 2013, Science, 340, 448

Atwood W. B. et al., 2009, ApJ, 697, 1071

NANOGrav Collaboration, Arzoumanian, Z., Brazier, A., et al. 2015, ApJ, 813, 65

Arzoumanian Z., et al., 2018, ApJS, 235, 37

Bailes, M., Barr, E., Bhat, N. D. R., et al. 2018, arXiv e-prints, arXiv:1803.07424

Bates S. D. et al., 2011, MNRAS, 416, 2455

Berezina, M., Champion, D. J., Freire, P. C. C., et al. 2017, MN-

RAS, 470, 4421

Blandford R., Teukolsky S. A., 1976, ApJ, 205, 580

Cordes, J. M., \& Lazio, T. J. W. 2002, arXiv e-prints, astro$\mathrm{ph} / 0207156$

Cromartie, H. T., Fonseca, E., Ransom, S. M., et al. 2019, Nature Astronomy, 439

Damour T., Deruelle N., 1986, Annales de L'Institut Henri

Poincaré, Phys. Theór., 44, 263

Damour T., Taylor J. H., 1992, Phys. Rev. D, 45, 1840
Demorest P. B., Pennucci T., Ransom S. M., Roberts M. S. E., Hessels J. W. T., 2010, Nature, 467, 1081

Demorest, P., Butler, B. J., Cordes, J. M., et al. 2015, American Astronomical Society Meeting Abstracts \#225 225, 346.01

Desvignes G., Barott W. C., Cognard I., Lespagnol P., Theureau G., 2011, in Burgay M., D’Amico N., Esposito P., Pellizzoni A., Possenti A., eds, American Institute of Physics Conference Series Vol. 1357 of American Institute of Physics Conference Series, A new pulsar instrumentation at the Allen Telescope Array and the Nancay Radio Telescope. pp 349-350

Edwards R. T., Hobbs G. B., Manchester R. N., 2006, MNRAS, 372, 1549

Folkner W. M., Williams J. G., Boggs D. H., 2009, Interplanetary

Network Progress Report, 178, C1

Freire P. C. C., Wex N., 2010, MNRAS, 409, 199

Granet C. et al., 2005, IEEE Antennas Propagation Magazine, 47, 13

Guillemot L., Tauris T. M., 2014, MNRAS, 439, 2033

Hobbs G. B., Edwards R. T., Manchester R. N., 2006, MNRAS, 369,655

Hotan A. W., van Straten W., Manchester R. N., 2004, PASA, 21, 302

Keith M. J. et al., 2010, MNRAS, 409, 619

Lam M. T., Cordes J. M., Chatterjee S., Dolch T., 2015, ApJ, 801, 130

Lange C., Camilo F., Wex N., Kramer M., Backer D. C., Lyne A. G., Doroshenko O., 2001, MNRAS, 326, 274

Lazarus P., Karuppusamy R., Graikou E., Caballero R. N., Champion D. J., Lee K. J., Verbiest J. P. W., Kramer M., 2016, MNRAS, 458, 868

Lorimer D. R., Kramer M., 2004, Handbook of Pulsar Astronomy Ng C. et al., 2014, MNRAS, 439, 1865

Özel F., Freire P., 2016, ARA\&A, 54, 401

Radhakrishnan V., Cooke D. J., 1969, Astrophys. Lett., 3, 225

Romani R. W., Watters K. P., 2010, ApJ, 714, 810

Shapiro I. I., 1964, Physical Review Letters, 13, 789

Shklovskii I. S., 1970, Soviet Ast., 13, 562

Splaver E. M., Nice D. J., Arzoumanian Z., Camilo F., Lyne A. G., Stairs I. H., 2002, ApJ, 581, 509

Stappers, B. W., Keane, E. F., Kramer, M., et al. 2018, Philosophical Transactions of the Royal Society of London Series A, 376, 20170293

Staveley-Smith L. et al., 1996, PASA, 13, 243

Tauris T. M., Savonije G. J., 1999, A\&A, 350, 928

Taylor J. H., 1992, Royal Society of London Philosophical Transactions Series A, 341, 117

Will C. M., 1993, Theory and Experiment in Gravitational Physics Yao, J. M., Manchester, R. N., \& Wang, N. 2017, ApJ, 835, 29 